\title{
Aquaporin evolution in fishes
}

\author{
Roderick Nigel Finn ${ }^{1,2} *$ and Joan Cerdà ${ }^{3}$ \\ 1 Institute of Biology, Bergen High Technology Center, University of Bergen, Bergen, Norway \\ ${ }^{2}$ Institute of Marine Research, Bergen, Norway \\ ${ }^{3}$ Laboratory of Institut de Recerca i Tecnologia Agroalimentàries, Institut de Ciències del Mar, Consejo Superior de Investigaciones Científicas, Barcelona, Spain
}

Edited by:

Steffen Madsen, University of Southern Denmark, Denmark

Reviewed by:

Steffen Madsen, University of Southern Denmark, Denmark Gordon Cramb, University of St Andrews, UK

Christian K. Tipsmark, University of

Arkansas, USA

${ }^{*}$ Correspondence:

Roderick Nigel Finn, Institute of

Biology, Bergen High Technology

Center, University of Bergen, PO Box

7803, N-5020 Bergen, Norway.

e-mail: nigel.finn@bio.uib.no
Aquaporins represent a primordial group of transmembrane solvent channels that have been documented throughout the living biota. This facet alone emphasizes the positive selection pressure for proteins associated with intracellular fluid homeostasis. Amongst extant Eukaryota the highest gene copy number can be found in plants and teleosts, a feature that reflects the genomic duplication history in both groups. In this minireview we discuss the discovery, structure, duplication, and diversification of the aquaporin superfamily. We focus on teleosts as the main models, but include data available for other organisms to provide a broader perspective.

\section{Keywords: aquaporin, gene duplication, evolution, neofunctionalization, water transport}

\section{INTRODUCTION}

Historically the transport of water across hydrophobic membranes was considered to occur via non-specific leakage. However, due to observations of increased water permeation in certain cells or tissues, such as human erythrocytes and the urinary bladder of frogs, specific water channels were also thought to exist (reviewed by Agre et al., 2001; Agre, 2005). The journey toward the discovery of aquaporins was aided by observations that junctional proteins were highly enriched in the plasma membrane of the bovine lens (Bloemendal et al., 1972; Broekhuyse and Kuhlmann, 1974). When purified and subjected to electrophoresis, such junctional complexes migrated as band III, MP26, or MP34 and were termed the main or major intrinsic protein (MIP) of the lens fiber (Broekhuyse et al., 1976; Vermorken et al., 1977; Gorin et al., 1984). However, it was by studying membranes purified from human erythrocytes that the first evidence of a bonafide water channel emerged (Benga et al., 1986a,b). The pure form of this $\sim 38-60 \mathrm{kDa}$ glycosylated channel was isolated and shown to be a non-glycosylated $28 \mathrm{kDa}$ MIP (CHIP28) of erythrocyte and renal membranes (Denker et al., 1988; Preston and Agre, 1991; Preston et al., 1992). These latter studies further provided direct evidence of water channel function through ex vivo injection of the CHIP28 transcript in Xenopus laevis oocytes followed by exposure of the oocytes to hyposmotic challenge. To concur with conventions established by the Human Genome Nomenclature Committee the term aquaporin-1 (AQP1) was coined for the CHIP28 protein (Agre et al., 1993) while MIP, which showed strong sequence homology to AQP1, became known as AQP0.

Since these early ground-breaking studies, aquaporins have been documented in all kingdoms of life, with several thousand sequences now available in public databases. The largest repertoire is currently found in plants with up to 71 paralogs reported in upland cotton (Park et al., 2010). In vertebrates the highest copy number is found in teleosts with up to 18 paralogs reported in zebrafish (Tingaud-Sequeira et al., 2010), while mammals run a close second due to tandem duplication of $A Q P 7$ and -12 resulting in up to 17 paralogs in humans (King et al., 2004; Cerdà and Finn, 2010). The precise copy number in Teleostei may increase as new genomes become available and novel annotations arise. For example the latest version of Ensembl (v63) predicts a second aqp $8 b$ paralog in zebrafish (ENSDARG00000089749) that is identical and closely linked to the aqp8b paralog (ENSDARG00000015512) characterized by Tingaud-Sequeira et al. (2010).

The vertebrate aquaporin complement has been classified according to molecular phylogeny, permeation preference for water, glycerol, or other small solutes and gases, tissue expression pattern and tertiary structure (Stahlberg et al., 2001; King et al., 2004; Takata et al., 2004; Zardoya, 2005; Tingaud-Sequeira et al., 2010). The superfamily thus consists of 13 subfamilies that include classical aquaporins (Aqp0, $-1,-2,-4,-5$, and -6), aquaglyceroporins (Aqp3, -7, -9, and -10), aquaporin-8 (Aqp8), and unorthodox aquaporins (Aqp11 and -12). A 14th subfamily suggested not to be present in mammals has also been identified in frogs ( $A Q P x l o$, Virkki et al., 2002). This gene is present in the Western-clawed frog genome (ENSXETG00000016307), but also exists in Prototheria (platypus: ENSOANG00000009732). Bayesian analysis of the amphibian and platypus sequences (data not shown) reveal that they are closely related to the aquaglyceroporins AQP3 and -9. To date, this gene has not been found in eutherian, metatherian, saurian, or piscine genomes.

\section{STRUCTURAL DIVERSITY AND EXPRESSION OF PISCINE AQUAPORINS}

By coupling the molecular phylogeny to linkage maps and comparing these data with structural and functional analyses it is 
becoming possible to establish parsimonious models of the duplication history of a given superfamily of genes. This approach was recently adopted for the aquaporin superfamily in zebrafish (Tingaud-Sequeira et al., 2010). The study revealed that Teleostei mostly retain two or in some cases three orthologs of the human aquaporin repertoire. The exceptions include AQP2, -4, -5, -6, -7 , and -12 , which are present in Teleostei as single copy genes, a putative pseudogene or are absent. Alignment of the zebrafish primary structures shows that they retain the canonical features of the human counterparts, including the six transmembrane $\alpha$-helices $(1,2,4,5,6$, and 8), five loops (A-E) and the two hemi-helices ( 3 and 7 ) that in most paralogs respectively retain the Asn-Pro-Ala (NPA) and Asn-Pro-Ala-Arg (NPAR) motifs that are the hallmark of the superfamily (Figure 1A). Comparison of the subdomains within each aquaporin group reveals that they have not experienced strong purifying selection. This is likely due to relaxed criteria associated with selection of amphipathic amino acids necessary for membrane integration. By contrast, hemi-helix 7 that bears the Arg-constriction residue shows the highest identity in all groups. This residue comprises one of a quartet that forms the aromatic-arginine ( $\mathrm{ar} / \mathrm{R})$ selectivity filter in the outer third of the channel vestibule (de Groot and Grubmüller, 2005; Wu and Beitz, 2007). We have previously reported that amino acid residues involved in the ar/ $\mathrm{R}$ constriction of teleost water channels are, like the mammalian counterparts, related to the different classes of aquaporins, but in Teleostei the underlying codons have experienced greater nucleotide substitution even in closely related paralogs (Cerdà and Finn, 2010). In some cases, this latter feature appears to have given rise to non-canonical residues associated with the selectivity filters. Examples include members of the teleost Aqp3a, -3b, -7, $-8 \mathrm{ab},-8 \mathrm{~b}$, and unorthodox aquaporins for the first NPA motif, and Aqp7, -8b, and the unorthodox aquaporins for the second NPA motif. Since there is a paucity of channel permeation preference studies in fishes (Fabra et al., 2006; Tingaud-Sequeira et al., 2008, 2010; MacIver et al., 2009; Hamdi et al., 2010; Chaube et al., 2011; Chauvigné et al., 2011), with a bias toward zebrafish, the functional and evolutionary significance of such core divergences remains to be determined. Interestingly, however, a recent study has revealed that the non-canonical Cys in the first NPA motif of murine AQP11 appears to augment oligomerization, suggesting that internal residues may play roles beyond permeation preference (Ikeda et al., 2011).

The regions of least conservation are the cytoplasmic $\mathrm{N}$ - and C-terminal domains. This is true both in terms of the number and identity of the encoded amino acids. The longest N-termini are present in teleost Aqp4, $-8 \mathrm{aa},-8 \mathrm{ab},-8 \mathrm{~b}$, and the unorthodox aquaporins, the structural features of which are also conserved in the mammalian orthologs. With respect to fish, the molecular role of the $\mathrm{N}$-terminus remains unknown. The role of this domain has, however, been associated with channel gating in plants (Törnroth-Horsefield et al., 2006), the intracellular localization of AQP6 (Beitz et al., 2006), and potentially the membrane trafficking and assembly of AQP4 variants in mammals (Neely et al., 1999). By contrast phosphorylation-mediated post-translational modifications of the C-terminal domain are well established for membrane trafficking and water permeability of mammalian AQP2 and amphibian AQP-h2/3 orthologs (Deen et al., 1994; Suzuki and Tanaka, 2009; Eto et al., 2011; Tamma et al., 2011). However, mutagenic studies addressing the role of this domain in teleost aquaporins has produced more divergent results (see below).

Expression data for piscine aquaporins have recently been reviewed by Cerdà and Finn (2010) and are schematically summarized here for zebrafish and other teleosts (Figure 1B). Three paralogs, Aqp laa, -3a, and -12, are expressed ubiquitously or semiubiquitously in the range of tissues that have been examined to date. With the exception of Aqp12, which differs markedly from the near-exclusive sites in mammals (Itoh et al., 2005), such ubiquitous expression patterns might indicate constitutive roles associated transcellular fluid transport. Conversely, the more restricted and partially redundant expression profiles of the other paralogs suggest that novel functions have or are evolving. As an example, Aqp0a and $-0 \mathrm{~b}$ are both considered necessary for normal lens development and transparency, as found for mammals, but Aqp0b is thought to have subfunctionalized (Froger et al., 2010). This latter study further suggested that teleost Aqp0b could be involved in cell adhesion, which is inline with the earliest studies in bovine lens and emerging evidence for other animal orthologs such as mammalian AQP4 (Hiraoki et al., 2006) and dipteran big brain (Tatsumi et al., 2009).

\section{DUPLICATION AND DUAL NEOFUNCTIONALIZATION OF PISCINE AQUAPORINS}

Within the last decade, investigations of piscine aquaporins have advanced from identification of the first ortholog in European eel (Aqp3b, Cutler and Cramb, 2000) to characterization of the superfamily in zebrafish (Tingaud-Sequeira et al., 2010). The latter study utilized the multidisciplinary approach outlined above and proposed a new nomenclature in order to provide a common platform for future research. The phylogenetic data set primarily included Teleostei, but also incorporated more ancestral aquaporins available for Hyperotreti, Chondrichthyes, and members of the Sarcopterygii. There remains a clear absence of data available for ancient piscine lineages such as Polypteriformes, Acipenseriformes, Semionotiformes, and Amiiformes. Such data would provide a more complete picture of aquaporin evolution in the actinopterygian lineage.

The current theory for the origin of the majority of aquaporins in Teleostei is consistent with an ancient whole genome duplication (WGD) event at the root of the crown clade (Amores et al., 1998; Jaillon et al., 2004; Volff, 2005; Finn and Kristoffersen, 2007; Tingaud-Sequeira et al., 2010). However, two subfamilies (aqp1 and aqp8) remained equivocal despite dichotomous and trichotomous clustering in relation to the human orthologs. The duplication history and putative neofunctionalization of the teleost aqp8aa, $-8 a b$, and $-8 b$ paralogs has been explained on the basis of a combination of WGD and tandem replication (Cerdà and Finn, 2010), while the aqp1 paralogs were suggested to have arisen via tandem duplication due to their juxtaposition in teleost genomes (Tingaud-Sequeira et al., 2008). The latter hypothesis, although seemingly parsimonious, did not incorporate the theory of diploidization and rearrangement or account for the loss of the WGD product. A new study has recently been conducted to address these issues (Zapater et al., 2011). Based upon extensive molecular phylogenetic, syntenic, and functional analyses, this 


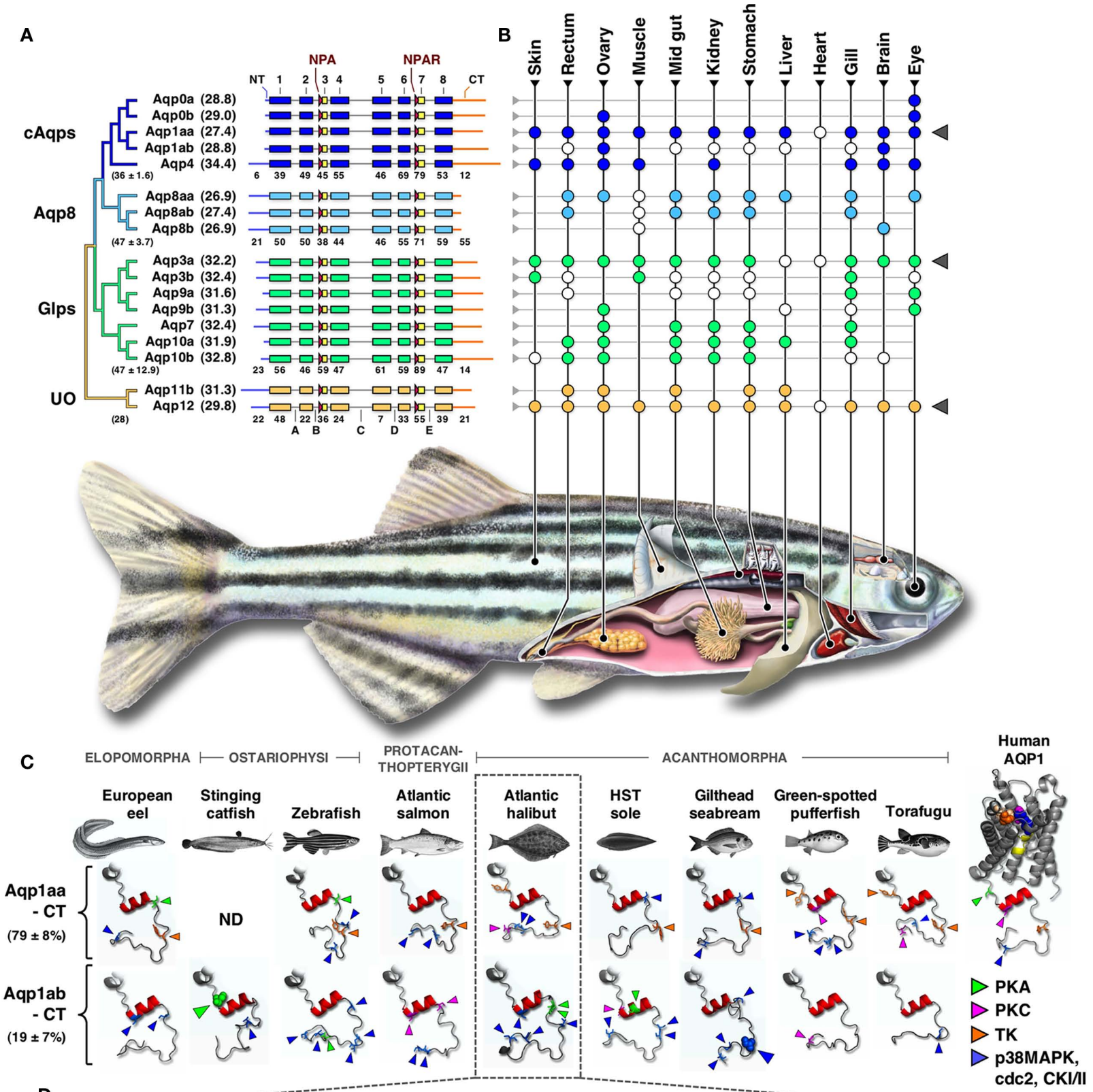

D
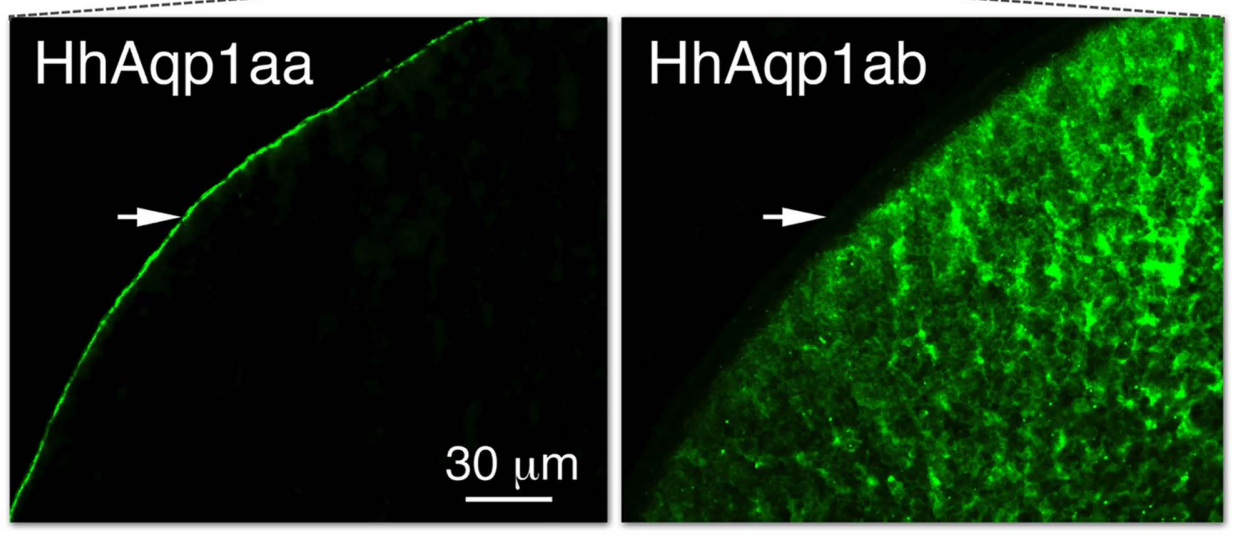

FIGURE 1 | Continued 
FIGURE 1 | Phylogeny, structure, and expression of teleost aquaporins.

(A) Bayesian midpoint rooted tree of the zebrafish aquaporin superfamily with predicted molecular masses of the deduced proteins given in parentheses. Linear-scale alignment of the superfamily shows the secondary structural conservation of $\alpha$-helices $1-8$ and the diversity of the N- (NT) and C-termini (CT) within each subfamily. The canonical Asn-Pro-Ala (NPA) and Asn-Pro-Ala-Arg (NPAR) motifs that are present in most paralogs are shown upstream of hemi-helices 3 and 7, respectively. Loops A-E are indicted below the unorthodox (UO) aquaporins. Identity values of the full-length (in parentheses \pm SD), the $\alpha$-helical, and the terminal subdomains of the classical aquaporins (cAqps), aquaporin 8 (Aqp8), aquaglyceroporins (Glps), and the $\mathrm{UO}$ aquaporins are shown below each group. (B) Summarized view of the tissue expression pattern of zebrafish aquaporins. Colored dots refer to the distribution in adults, while white dots represent data reported for other species of teleost. In some species the anterior intestine is indicated by the stomach, while the brain includes chemosensory and mechanosensory organs. Gray triangles to the right highlight ubiquitous or semi-ubiquitous expression patterns. (C) Three-dimensional cartoon renders of the cytoplasmic C-terminal regions of teleost Aqp1aa and -1ab compared to
Human AQP1 (1 H6I). Models were generated using ModWeb and rendered with MacPyMOL. For the human AQP1 channel, the NPA motifs are highlighted in yellow and the ar/R constriction residues (Phe, magenta; His, wheat; Cys, Orange; Arg, blue) are shown as spacefill. All proteins retain a tertiary helix (red $\mathrm{H} 9$ ) but otherwise fold as disordered loops extending intracellularly from $\alpha$-helix 8 . Putative phosphorylation sites are indicated as protein kinase $A(P K A)$, protein kinase $C$ (PKC), tyrosine kinase (TK), p38 mitogen-activated protein kinase (p38MAPK), cell division cycle 2 (cdc2), or casein type kinase (CKI/II). Phosphorylation sites known to influence membrane trafficking are rendered as spacefill for the stinging catfish (green) and gilthead seabream (blue). Identity $( \pm S D$ ) of the aligned teleost C-termini are given to the left. ND: no data. (D). Localized cellular expression of Atlantic halibut (Hippoglossus hippoglossus) HhAqp1aa and -1ab (green) following ex vivo injection of transcripts in Xenopus laevis oocytes. HhAqp1aa is constitutively expressed in the plasma membrane (white arrows), while HhAqp1ab is retained in intracellular vesicles. Data for this figure are recompiled from Cerdà and Finn (2010), Sun et al. (2010), Tingaud-Sequeira et al. (2008, 2010), Tipsmark et al. (2010), Chaube et al. (2011), Zapater et al. (2011), and Zichichi et al. (2011). latter study concluded that the teleost aqp1 genes (formerly annotated as aqp $1 a$ and $-1 b$ ) are indeed tandem duplicates and were respectively renamed $a q p 1 a a$ and $a q p 1 a b$ to match the $a q p 8$ terminology, while the $a q p 5 / 1 b$ pseudogene in zebrafish is the most likely candidate for the lost WGD product.

The study by Zapater et al. (2011) further revealed that the cytoplasmic C-terminus of Aqplab is the most rapidly evolving subdomain in the vertebrate aquaporin superfamily (Figure 1C). Despite this degeneracy, Aqplab has been found to play a conserved role in the oocyte hydration of teleosts (reviewed by Cerdà, 2009; Finn and Fyhn, 2010). The novel role of Aqp1ab in oocyte hydration was first suggested for gilthead seabream (Fabra et al., 2005), and has recently been experimentally demonstrated in the acanthomorph teleost Atlantic halibut (Zapater et al., 2011). The data for Atlantic halibut show that the temporal insertion of the Aqplab paralog in the oocyte plasma membrane occurs during the phase of maximal osmolyte generation (Finn et al., 2002; Finn, 2007; Zapater et al., 2011) and differs markedly from the predominant thecal localization of AQP1 in pre-ovulatory stages of human follicles (Thoroddsen et al., 2011). An interesting aspect of the role of Aqp1ab in teleost oocytes is that it essentially functions as an inwardly rectifying channel due to the exclusive generation of intracellular osmolytes and the tight temporal regulation of the channel in the oolemma. Perhaps even more striking is that the rapid evolution of the Aqplab C-terminus has resulted in alternative control of the trafficking mechanism and in some species, such as the Atlantic halibut, loss of function when expressed in $X$. laevis oocytes (Figure 1D). It has been suggested that phosphorylation of $\mathrm{Ser}^{227}$ is associated with translocation of stinging catfish Aqp1ab (Chaube et al., 2011), while phosphorylation of $\mathrm{Ser}^{254}$ promotes recycling of gilthead seabream Aqp lab (TingaudSequeira et al., 2008), yet phosphorylation may not be involved in the trafficking mechanism of Atlantic halibut Aqplab (Zapater

\section{REFERENCES}

Agre, P. (2005). Aquaporin water channels. Biosci. Rep. 24, 127-163.

Agre, P., Borgnia, M. J., Yasui, M., Neely,

J. D., Carbrey, J., Kozono, D., Beitz,

E., Hoffert, J., Leitch, V., and King,
L. S. (2001). "Discovery of the aquaporins and their impact on basic and clinical physiology," in Aquaporins, eds S. Hohmann, S. Nielsen, and P. Agre (New York: Academic Press), $1-39$.

et al., 2011). These findings are revealing that for certain paralogs, gene duplication has resulted in constrained and relaxed traits controlling aquaporin function in teleosts. For the Aqplab paralogs, selection pressure has favored oocyte hydration, but has been relaxed with regard to the molecular control of membrane trafficking. The nature of these trafficking mechanisms and the intracellular pathways involved are not yet known, and remain intriguing avenues for future research.

\section{FUTURE PERSPECTIVES}

Investigations of the role of aquaporins in fishes are only just beginning. By comparing the results obtained from fishes to the more extensive data sets available for mammals, it seems likely that rapid advances can be achieved. From an evolutionary perspective, however, the piscine branch of vertebrates includes members that existed both prior to and after the second, third, and in some species of teleost the fourth round of WGD. Consequently by studying the superfamily in selected organisms, it should be possible to determine how gene duplication or gene loss has resulted in alternative evolution of aquaporins at the molecular, cellular, physiological, and ecological levels. Today, the number of extant vertebrates $(\sim 55,000)$ is essentially equally split between aerial and aquatic environments. It can be expected that selection pressure has acted differently in these ecosystems.

\section{ACKNOWLEDGMENTS}

The authors gratefully acknowledge financial support provided by Grants from the Research Council of Norway (204813) to Roderick Nigel Finn and the Spanish Ministry of Science and Innovation (MICINN; AGL2007-60261 and AGL2010-15597) and European Commission (MRTN-CT-2006-035995-Aquaglyceroporins) to Joan Cerdà. The authors further wish to thank Jón Baldur Hlidberg for artwork.

Agre, P., Sasaki, S., and Chrispeels, M. J. (1993). Aquaporins, a family of membrane water channel proteins. Am. J. Physiol. 265, F461.

Amores, A., Force, A., Yan, Y. L., Joly, L., Amemiya, C., Fritz, A., Ho,
R. K., Langeland, J., Prince, V., Wang, Y. L., Westerfield, M., Ekker, M., and Postlethwait, J. H. (1998). Zebrafish hox clusters and vertebrate genome evolution. Science 282, 1711-1714. 
Beitz, E., Liu, K., Ikeda, M., Guggino, W. B., Agre, P., and Yasui, M. (2006). Determinants of AQP6 trafficking to intracellular sites versus the plasma membrane in transfected mammalian cells. Biol. Cell 98, 101-109.

Benga, G., Popescu, O., Borza, V., Pop, V. I., Muresan, A., Mocsy, I., Brain, A., and Wrigglesworth, J. M. (1986a). Water permeability of human erythrocytes. Identification of membrane proteins involved in water transport. Eur. J. Cell Biol. 1986, 41, 252-262.

Benga, G., Popescu, O., Borza, V., Pop, V. I., and Holmes, R. E. (1986b). Chloromercuribenzenesulfonate binding by membrane proteins and the inhibition of water transport in human erythrocytes. Biochemistry 25, 1535-1538.

Bloemendal, H., Vermorken, Z. F., Dunia, I., and Benedetti, E. L. (1972). The plasma membranes of eye lens fibres. Biochemical and structural characterization. Cell Differ. 1, 91-106.

Broekhuyse, R. M., and Kuhlmann, F. D. (1974). Lens membranes 1. Composition of urea-treated plasma membranes from calf lens. Exp. Eye Res. 19, 297-302.

Broekhuyse, R. M., Kuhlmann, F. D., and Stols, L. H. (1976). Lens membranes II. Isolation and characterization of the main intrinsic polypeptide (MIP) of bovine lens fiber membranes. Exp. Eye Res. 23, 365-371.

Cerdà, J. (2009). Molecular pathways during marine fish egg hydration: the role of aquaporins. J. Fish Biol. 75, 2175-2196.

Cerdà, J., and Finn, R. N. (2010). Piscine aquaporins: an overview of recent advances. J. Exp. Zool. 313A, 623-650.

Chaube, R., Chauvigné, F., TingaudSequeira, A., Joy, K. P., Acharjee, A., Singh, V., and Cerdà, J. (2011). Molecular and functional characterization of catfish (Heteropneustes fossilis) aquaporin-1b: changes in expression during ovarian development and hormoneinduced follicular maturation. Gen. Comp. Endocrinol. 170, 162-171.

Chauvigné, F., Lubzens, E., and Cerdà, J. (2011). Design and characterization of genetically engineered zebrafish aquaporin-3 mutants highly permeable to the cryoprotectant ethylene glycol. BMC Biotechnol. 11, 34. doi: 10.1186/1472-675011-34

Cutler, C. P., and Cramb, G. (2000). "Water transport and aquaporin expression in fish," in Molecular Biology and Physiology of Water and
Solute Transport, eds S. Hohmann and S. N. Neilsen (New York: Kluwer Academic/Plenum Publishers), 433-441.

de Groot, B. L., and Grubmüller, H. (2005). The dynamics and energetics of water permeation and proton exclusion in aquaporins. Curr. Opin. Struct. Biol. 15, 176-183.

Deen, P. M. T., Verdijk, D. M. A., Knoers, N. V. A. A., Wieringa, B., Monnens, L. A. H., van Os, C. H., and van Oost, B. A. (1994). Requirement of human renal water channel aquaporin-2 for vasopressindependent concentration of urine. Science 264, 92-95.

Denker, B. M., Smith, B. L., Kuhajda, F. P., and Agre, P. (1988). Identification, purification, and partial characterization of a novel Mr 28,000 integral membrane protein from erythrocytes and renal tubules. J. Biol. Chem. 263, 15634-15642.

Eto, K., Noda, Y., Horikawa, S., Uchida, S., and Sasaki, S. (2011). Phosphorylation of aquaporin-2 regulates its water permeability. J. Biol. Chem. 285, 40777-40784.

Fabra, M., Raldúa, D., Bozzo, M. G., Deen, P. M. T., Lubzens, E., and Cerdà, J. (2006). Yolk proteolysis and aquaporin-1o play essential roles to regulate fish oocyte hydration during meiosis resumption. Dev. Biol. 295, 250-262.

Fabra, M., Raldúa, D., Power, D. M., Deen, P. M. T., and Cerdà, J. (2005). Marine fish egg hydration is aquaporin-mediated. Science 307, 545

Finn, R. N. (2007). The maturational disassembly and differential proteolysis of paralogous vitellogenins in a marine pelagophil teleost: a conserved mechanism of oocyte hydration. Biol. Reprod. 76, 936-948.

Finn, R. N., and Fyhn, H. J. (2010). Requirement for amino acids in ontogeny of fish. Aquac. Res. 41, 684-716.

Finn, R. N., and Kristoffersen, B. A. (2007). Vertebrate vitellogenin gene duplication in relation to the "3R hypothesis": correlation to the pelagic egg and the oceanic radiation of teleosts. PLoS ONE 2, e169. doi: 10.1371/journal.pone.0000169

Finn, R. N., Østby, G. C., Norberg, B., and Fyhn, H. J. (2002). In vivo oocyte hydration in Atlantic halibut (Hippoglossus hippoglossus): proteolytic liberation of free amino acids, and ion transport, are driving forces for osmotic water influx. J. Exp. Biol. 205, 211-224.

Froger, A., Clemens, D., Kalman, K., Németh-Cahalan, K.-L., Schilling, T. F., and Hall, J. E. (2010). Two distinct aquaporin 0s are required for development and transparency of the zebrafish Lens. Invest. Ophthalmol. Vis. Sci. 51, 6582-6589.

Gorin, M. B., Yancey, S. B., Cline, J., Revel, J. P., and Horwitz, J. (1984). The major intrinsic protein (MIP) of the bovine lens fiber membrane: characterization and structure based on cDNA cloning. Cell 39, 49-59.

Hamdi, M., Sanchez, M. A., Beene, L. C., Liu, Q., Landfear, S. M., Rosen, B. P., and Liu, Z. (2010). Arsenic transport by zebrafish aquaglyceroporins. BMC Mol. Biol. 10, 104. doi: 10.1186/1471-2199-10-104

Hiraoki, Y., Tani, K., Kamagawa, A., Gyobu, N., Nishikawa, K., Suzuki, H., Walz, T., Sasaki, S., Mitsuoki, K., Kimura, K., Mizoguchi, A., and Fujiyoshi, Y. (2006). Implications of the aquaporin-4 structure on array formation and cell adhesion. J. Mol. Biol. 355, 628-639.

Ikeda, M., Andoo, A., Shimono, M., Takamatsu, N., Taki, A., Muta, K., Matsushita, W., Uechi, T., Matsuzaki, T., Kenmochi, N., Takata, K., Sasaki, S., Ito, K., and Ishibasji, K. (2011). The NPC motif of aquaporin-11, unlike the NPA motif of known aquaporins, is essential for full expression of molecular function. J. Biol. Chem. 286, 3342-3350.

Itoh, T., Rai, T., Kuwahara, M., Ko, S. B., Uchida, S., Sasaki, S., and snd Ishibashi, K. (2005). Identification of a novel aquaporin, AQP12, expressed in pancreatic acinar cells. Biochem. Biophys. Res. Commun. 330, 832-838.

Jaillon, O., Aury, J. M., Brunet, F., Petit, J. L., Stange-Thomann, N., Mauceli, E., Bouneau, L., Fischer, C., Ozouf-Costaz, C., Bernot, A., Nicaud, S., Jaffe, D., Fisher, S., Lutfalla, G., Dossat, C., Segurens, B., Dasilva, C., Salanoubat, M. Levy, M., Boudet, N., Castellano, S., Anthouard, V., Jubin, C., Castelli, V., Katinka, M., Vacherie, B., Biémont, C., Skalli, Z., Cattolico, L., Poulain, J., De Berardinis, V., Cruaud, C., Duprat, S., Brottier, P., Coutanceau, J. P., Gouzy, J., Parra, G., Lardier, G., Chapple, C., McKernan, K. J., McEwan, P., Bosak, S., Kellis, M., Volff, J. N., Guigó, R., Zody, M. C., Mesirov, J., Lindblad-Toh, K., Birren, B. Nusbaum, C., Kahn, D., RobinsonRechavi, M., Laudet, V., Schachter, V., Quétier, F., Saurin, W., Scarpelli, C., Wincker, P., Lander, E. S., Weissenbach, J., and Roest Crollius, H. (2004). Genome duplication in the teleost fish Tetraodon nigroviridis reveals the early vertebrate proto-karyotype. Nature 431, 946-957.

King, L. S., Kozono, D., and Agre, P. (2004). From structure to disease: the evolving tale of aquaporin biology. Nat. Rev. Mol. Cell Biol. 5, 687-698.

MacIver, B., Cutler, C. P., Yin, J., Hill, M. G., Zeidel, M. L., and Hill, W. G. (2009). Expression and functional characterization of four aquaporin water channels from the European eel (Anguilla anguilla). J. Exp. Biol. 212, 2856-2863.

Neely, J. D., Christensen, B. M., Nielsen, S., and Agre, P. (1999). Heterotetrameric composition of aquaporin4 water channels. Biochemistry 38 , 11156-11163.

Park, W., Scheffler, B. E., Bauer, P. J., and Campbell, P. T. (2010). Identification of the family of aquaporin genes and their expression in upland cotton (Gossypium hirsutum L.). BMC Plant Biol. 10, 142. doi 10.1186/1471-2229-10-142

Preston, G. M., and Agre, P. (1991). Isolation of the cDNA for erythrocyte integral membrane protein of 28 kilodaltons: member of an ancient channel family. Proc. Natl. Acad. Sci. U.S.A. 88, 11110-11114.

Preston, G. M., Carroll, T. P., Guggino, W. B., and Agre, P. (1992). Appearance of water channels in Xenopus oocytes expressing red cell CHIP28 protein. Science 256, 385-387.

Stahlberg, H., Heymann, B., Mitsuoka, K., Fuyijoshi, Y., and Engel, A. (2001). "The aquaporin superfamily: structure and function," in Aquaporins, eds S. Hohmann, S. Nielsen, and P. Agre (New York: Academic Press), 40-119.

Sun, Y., Zhang, Q., Qi, J., Chen, Y., Zhong, Q., Li, C., Yu, Y., Li, S., and Wang, Z. (2010). Identification of differential genes in the ovary relative to the testis and their expression patterns in half-smooth tongue sole (Cynoglossus semilaevis). J. Genet. Genomics 37, 137-145.

Suzuki, M., and Tanaka, S. (2009). Molecular and cellular regulation of water homeostasis in anuran amphibians by aquaporins. Comp. Biochem. Physiol. 153A: 231-241.

Takata, K., Matsuzaki, T., and Tajika, Y. (2004). Aquaporins: water channel proteins of the cell membrane. Prog. Histochem. Cytochem. 39, 1-83.

Tamma, G., Robben, J. H., Trimpert, C., Boon, M., and Deen, P. M. T. (2011). Regulation of AQP2 localization by S256 and S261 phosphorylation and ubiquitination. Am. J. Physiol., Cell Physiol. 300, C636-C646. 
Tatsumi, K., Tsuji, S., Miwa, H., Morisaku, T., Nuriya, M., Orihara, M., Kaneko, K., Okano, H., and Yasui, M. (2009). Drosophila big brain does not act as a water channel, but mediates cell adhesion. FEBS Lett. 583, 2077-2082.

Thoroddsen, A., Dahm-Kähler, P., Lind, A. K., Weijdegård, B., Lindenthal, B., Müller, J., and Bränstrøm, M. (2011). The water permeability channels aquaporins 1-4 are differentially expressed in granulosa and theca cells of the preovulatory follicle during precise stages of human ovulation. J. Clin. Endocrinol. Metab. 96, 1021-1028.

Tingaud-Sequeira, A., Calusinska, M., Finn, R. N., Chauvigné, F., Lozano, J., and Cerdà, J. (2010). The zebrafish genome encodes the largest vertebrate repertoire of functional aquaporins with dual paralogy and substrate specificities similar to mammals. BMC Evol. Biol. 10, 38. doi: 10.1186/1471-2148-10-38

Tingaud-Sequeira, A., Chauvigné, F., Fabra, M., Lozano, J., Raldúa,
D., and Cerdà, J. (2008). Structural and functional divergence of two fish aquaporin-1 water channels following teleost-specific gene duplication. BMC Evol. Biol. 8, 259. doi: 10.1186/1471-21488-259

Tipsmark, C. K., Sørensen, K. J., and Madsen, S. S. (2010). Aquaporin expression dynamics in osmoregulatory tissues of Atlantic salmon during smoltification and seawater acclimation. J. Exp. Biol. 213, 368-379.

Törnroth-Horsefield, S., Wang, Y., Hedfalk, K., Johanson, U., Karlsson, M., Tajkhorshid, E., Neutze, R., and Kjellbom, P. (2006). Structural mechanism of plant aquaporin gating. Nature 439, 688-694.

Vermorken, A. J. M., Hildernink, J. M. H. C., Benedetti, E. L., and Bloemendal, H. (1977). Changes in membrane protein pattern in relation to lens cell differentiation. FEBS Lett. 83, 301-306.

Virkki, L. V., Franke, C., Somieski, P., and Boron, W. F. (2002). Cloning and functional characterization of a novel aquaporin from Xenopus laevis oocytes. J. Biol. Chem. 277, 40610-40616.

Volff, J. N. (2005). Genome evolution and biodiversity in teleost fish. Heredity 94, 280-294.

Wu, B., and Beitz, E. (2007). Aquaporins with selectivity for unconventional permeants. Cell. Mol. Life Sci. 64, 2413-2421.

Zapater, C., Chauvigné, F., Norberg, B., Finn, R. N., and Cerdà, J. (2011). Dual neofunctionalization of a rapidly evolving aquaporin1 paralog reveals constrained and relaxed traits controlling channel function during meiosis resumption in marine teleosts. Mol. Biol. Evol. doi: 10.1093/molbev/msr146. [Epub ahead of print].

Zardoya, R. (2005). Phylogeny and evolution of the major intrinsic protein family. Biol. Cell 97, 397-414.

Zichichi, R., Magnoli, D., Montalbano, G., Laurà, R., Vega, J. A., Ciriaco, E., and Germanà, A. (2011). Aquaporin 4 in the sensory organs of adult zebrafish (Danio rerio). Brain Res. 1384, 23-28.

Conflict of Interest Statement: The authors declare that the research was conducted in the absence of any commercial or financial relationships that could be construed as a potential conflict of interest.

Received: 24 May 2011; paper pending published: 29 June 2011; accepted: 16 July 2011; published online: 26 July 2011.

Citation: Finn $R N$ and Cerdà J (2011) Aquaporin evolution in fishes. Front. Physio. 2:44. doi: 10.3389/fphys.2011.00044

This article was submitted to Frontiers in Aquatic Physiology, a specialty of Frontiers in Physiology.

Copyright () 2011 Finn and Cerdà. This is an open-access article subject to a nonexclusive license between the authors and Frontiers Media SA, which permits use, distribution and reproduction in other forums, provided the original authors and source are credited and other Frontiers conditions are complied with. 